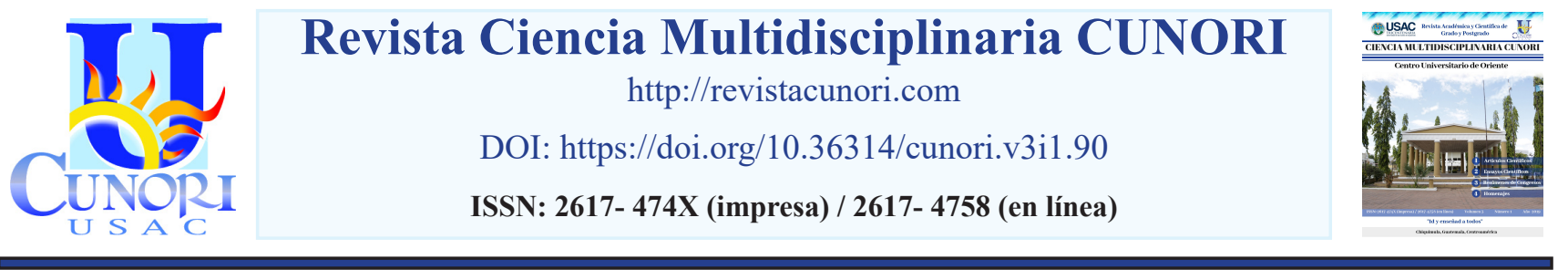

Como citar el ensayo

Chicas, R., Villela, C., (2019). Incidencia de la psicología en el planeamiento de la educación andragógica. Revista Ciencia Multidisciplinaria CUNORI, 3(1), 143-147. DOI: https://doi.org/10.36314/cunori.v3i1.90

\title{
Incidencia de la psicología en el planeamiento de la educación andragógica
}

\section{Incidence of psychology in the planning of andragogic education}

\author{
Regina Chicas* \& Claudia Villela \\ Departamento de Estudios de Posgrado, Centro Universitario de Suroriente -CUNSURORI- USAC. Jalapa \\ Recibido: 26 de noviembre de 2018 / Revisión: 14 de diciembre de 2018 / Aceptado: 02 de marzo de 2019
}

Disponible en internet el 30 de agosto de 2019

*Autor para correspondencia.

Correo electrónico: reginachicas@hotmail.com

Resumen

C e conoce como planificación generalmente en países de habla hispana, pero en el sentido más universal, implica tener uno o va$\checkmark$ rios objetivos en común, junto con las acciones necesarias para concluir con éxito. Otras definiciones, más precisas, incluyen la planificación como un proceso de toma de decisiones para alcanzar un futuro deseado, teniendo en cuenta la situación actual y los factores internos y externos que pueden influir en el logro de los objetivos, pasando de lo más simple a lo complejo, dependiendo de el medio a aplicar. La acción de planificación en la gestión se refiere a los planes y proyectos en sus diferentes áreas, niveles y actitudes. Se entiende por planificación y sus sinónimos, a cualquier actividad previamente programada para su realización o aplicación, siendo un proceso de suma importancia en todos los procesos de la vida, para obtener resultados positivos y lograr el éxito en el futuro. El objetivo del ensayo es analizar la importancia de la planificación, tanto en el campo universal como en el educativo.

Palabras clave: planeamiento, educación

Abstract

T $\mathrm{t}$ is known as planning, planning or planning, generally in Spanish-speaking countries, but in the most universal sense, it implies Lhaving one or several objectives in common, together with actions required to conclude successfully. Other definitions, more precise, include planning as a decision-making process to reach a desired future, taking into account the current situation and the internal and external factors that can influence the achievement of objectives, going from the simplest to the complex, depending on the medium to be applied. The action of planning in management refers to plans and projects in their different areas, levels and attitudes. It is understood by planning and its synonyms, to any activity previously programmed to its realization or application, being a process of utmost importance in all life processes, to obtain positive results and achieve success in the future. The aim of the essay is to analyze the importance of planning, both in the universal and educational field.

Keywords: planning, education 


\section{Introducción}

Es necesario comprender que previo a iniciar cualquier actividad es necesario contar con un plan, el cual debe ser objetivo, realista y claro, determinando el ¿Quién lo hará?, el ¿cómo se realizará?, el ¿cuándo?, el ¿por qué?, el ¿dónde?, con ¿qué recursos se realizará?, ¿qué resultados se esperan obtener?, ¿cómo será evaluado?

En el caso de la educación andragógica, la psicología tiene una gran incidencia debido a que se trata de enseñar a seres humanos, con diversidad de pensamientos, experiencias, contextos, con múltiples tipos de inteligencias y aprendizajes, es por ello que la forma de enseñarles tiene que ser también diferente. El maestro cambia su rol de ser un transmisor de conocimiento a ser un facilitador del aprendizaje, se cambia del proceso vertical al horizontal, en el cual el protagonista es el estudiante quien con su riqueza de conocimiento previo se le da oportunidad para exprese sus ideas y emociones. En el ensayo se pretende analizar la incidencia de la psicología en el planeamiento de la educación andragógica, tomando en cuenta los diversos factores.

\section{Factores incidentes en el aprendizaje de los adultos para ser incluidos en el planeamiento educativo}

Para tener claros los conceptos diremos que, la andragogía es la disciplina que se ocupa de la educación y aprendizaje del adulto. Etimológicamente la palabra adulto, proviene de la voz latina "adultus", que puede interpretarse como "ha crecido" luego de la etapa de la adolescencia. El crecimiento biológico del ser humano llega en un momento determinado al alcanzar su máximo desarrollo en sus aspectos fisiológicos,

Para Knowles et al., (2001), la andragogía ofrece los principios fundamentales que permiten el diseño y conducción de procesos docentes más eficaces, en el sentido que remite a las características de la situación de aprendizaje, y por tanto, es aplicable a diversos contextos de enseñanza de adultos, como por ejemplo: la educación comunitaria, el desarrollo de recursos humanos en las organizaciones y la educación universitaria.

Brandt (1998:48), sin embargo, en su intento de realizar una nueva conceptualización del término, señala que: "la andragogía se encarga de la educación entre, para y por adultos y la asume como su objeto de estudio y realización. Vista o concebida ésta, como autoeducación, es decir, interpreta el hecho educativo como un hecho andragógico, donde la educación se realiza en forma autónoma. Entendiéndose el proceso, mediante el cual, el ser humano consciente de sus posibilidades de realización, libremente selecciona, exige, asume el compromiso, con responsabilidad, lealtad y, sinceridad, de su propia formación y realización personal".

A diferencia del niño, el adulto como individuo maduro, manifiesta las siguientes características: Tiene un auto concepto, experiencia, prisa en aprender, orientación para el aprendizaje y motivación para aprender, se recordará que la educación de adultos, puede darse tanto en la educación formal, en la informal y en la no formal. 
Se pretende a través del presente ensayo determinar ¿de qué forma inciden los factores aprendizaje en la educación de adultos (los de adultez temprana, adultez media y adultez tardía)?. Existen algunas factores que inciden en gran medida, algunos de ellos son: la personalidad del estudiante adulto, su inteligencia o coeficiente intelectual, su percepción de las cosas, el grado de madurez que posee, la motivación que lo lleva a querer seguir preparándose, su capacidad de memorizar nueva información.

El docente, facilitador o tutor, debe tomar en cuenta los distintos tipos de personalidades de los estudiantes, debido a que en una misma aula pueden existir participantes extrovertidos e introvertidos, algunos muy participativos y otros aislados, por lo que se deberá utilizar una variedad de técnicas y estrategias para fomentar que se dé el proceso de construcción del conocimiento. En relación con el factor inteligencia, se puede determinar que existen factores genéticos, culturales, de oportunidad, etc. Que afectan al estudiante adulto, sin embargo la inteligencia la podemos definir de la siguiente forma: Spearman (1927), sostenía que es más fácil medir la inteligencia que definirla. Además, existen otros dos tipos de inteligencias: la abstracta, habilidad para manejar ideas, y la mecánica, habilidad para entender y manejar objetos.

Por otro lado, las diversas percepciones de los estudiantes, influyen grandemente en cómo se lleva el proceso de descubrimiento y construcción del mismo, lo que se comprende debido a que, las personas que se han criado en diferentes culturas aprenden de niños, sin que jamás se den cuenta de ello, al excluir cierto tipo de información, al mismo tiempo que atienden cuidadosamente a información de otra clase. Una vez instituidas, esas normas de percepción parecen seguir perfectamente invariables toda la vida. (Hall, 1983: 60-61). Por lo que la percepción es un proceso inconsciente que se desarrolla a lo largo de la vida y permite que el ser humano de forma automática asocie características, situaciones, objetos, etc. e intente acoplar la nueva información con sucesos de su pasado.

¿Qué es la madurez? de acuerdo con (Allport, 1961) que señala que la maduración es en realidad diferenciación e integración, tomamos estos dos conceptos que se adaptan al criterio recién establecido para delimitar así los objetivos y el proceso mismo de la maduración. La diferenciación significa la especialización de las capacidades y estructuras psicofísicas para determinada función cada vez más progresiva, cada vez más específica. Como lo señala el recién citado autor, el todo pre-estructurado se va diferenciando de acuerdo a las necesidades del organismo en el medio.

También el grado de madurez que tengan los estudiantes incidirá en su educación, hay que recordar que el crecimiento fisiológico no necesariamente va acompañado de la madures para la toma de decisiones, pero hay algo muy relevante de los adultos es su motivación por aprender, como se ha dicho el estudiante adulto se encuentra motivado a aprender por dos razones principales, la primera porque lo aplicará de inmediato y segundo lo hace por necesidad. Como resumen se puede decir que existen factores que se repiten en ellos y permite clasificarlos de acuerdo a sus personalidades, motivaciones, inteligencia y demás, por lo que los facilitadores deben realizar su rol, volviéndolos transformadores, cambiando el paradigma de que el catedrático todo lo sabe y es quien manda, pasando una educación activa, una educación horizontal, donde los alumnos aprenden del maestro pero a la vez, el maestro aprende del estudiante, a través de procesos de descubrimiento. 
Por lo que, considerando las características particulares de la madurez, los rasgos definidores del aprendizaje en la edad adulta y la posibilidad de desarrollo en las últimas etapas del ciclo vital, podemos concluir que la motivación es un constructo que sirve de conector entre las posibilidades de aprender y los objetivos de aprendizaje en las personas mayores.

¿Por qué es necesario tomar en consideración los factores psicológicos en el planeamiento de aprendizaje?, así como en la vida diaria diseñamos y realizamos algoritmos para solucionar los problemas cotidianos, es así que al levantarnos de la cama ya tenemos en la mente una serie de pasos que debemos seguir para llegar a la escuela o al trabajo. Una vez que nos encontramos en el lugar a donde se quería llegar, tenemos en nuestra mente una serie de tareas que debemos realizar en horarios ya establecidos.

El tema de algoritmos es muy interesante, ya que proporciona una serie de posibilidades para llevar a los estudiantes a un aprendizaje significativo. La enseñanza y el aprendizaje aportan habilidades cognitivas que mejoran el razonamiento, que conduce al estudiante a descubrir, crear e inventar, los medios que le permiten seguir con los procesos para la resolución de problemas en clase, y que a partir de este conocimiento puede contar con herramientas para solucionar problemas de la vida diaria.

Al momento de desarrollar un planeamiento andragógico de un curso, se debe recordar que el profesor debe asumir el rol de facilitador, permitiendo la relación entre los estudiantes de compartir sus conocimientos previos y los nuevos proporcionados para generar así el nuevo o los nuevos conocimientos, adicionalmente a ello el planeamiento debe hacerse tomando en cuenta los factores que inciden en el aprendizaje, como por ejemplo en aprendizaje cultural que proponía Vigotsky, donde se aprende del grupo, o de la forma de aprender como lo mostraba en su teoría Gardner con los tipos de inteligencias, recordemos que no todos aprenden de la misma forma ni al mismo ritmo, por lo que el planeamiento andragógico permite adecuar el aprendizaje a la diversidad del grupo. Se busca solucionar problemas específicos, que, al desarrollarlos, pongan en juego el pensamiento crítico, el análisis y el raciocinio, características con las que cuentan muy desarrolladas los estudiantes adultos, debido a que esas tres funciones se encuentran almacenadas en el Lóbulo Frontal del Cerebro el cual se termina de desarrollar completamente a los 20 años de edad.

Considerando la peculiaridad del público en cuestión y teniendo las afirmaciones de Kelvin Miller (Cavalcanti, 1999), que coloca en sus estudios que el estudiante adulto sólo consigue retener 10\% de la información que oye en una clase, después de 72 horas de recibida la información. En cambio, esto sostiene que este "retendrá hasta $85 \%$ de la información si además de oír, ver y hacer en seguida una actividad relacionada con la misma". Es importante recordar que los estudiantes andragógicos tienen prioridades en la vida y en la última es estudiar, es por ello que las planificaciones deben ser las más flexibles posibles.

\section{Conclusión}

Se puede concluir luego de los puntos expuestos que la psicología incide positivamente en el desarrollo del planeamiento de la educación andragógica debido a que permite analizar a cada grupo de estudiantes como personas individuales que tienen sus propias características y necesidades; así mismo que cada proceso de educación andragógica requiere indispensablemente de una planeación formativa flexible, 
que permita cada asignación, cada lectura, cada aprendizaje, satisfacer la necesidad del estudiante de aplicabilidad inmediata, y que debe ubicarse en un contexto de la realidad donde pueda ser ejemplificado y estudiado con características reales, claras y objetivas.

\section{Referencias bibliográficas}

Allport, G. W. (1961). Patrón y el crecimiento de la personalidad. New York: Holt, RineHard y Winston . Brandt, J. (1998). Andragogía: propuesta de autoeducación. Los Teques, Venezuela: Tercer Milenium.

Cavalcanti, M. C. (1999a) A práctica como fuente de proyectos de pesquisa para a formación de profesores. In: J. C. P. ALMEIDA F ${ }^{\mathrm{o}}$ (org.) Profesores de Lengua Extranjera em Formación. Campinas, SP: Editora Pontes.

Hall, E. T. (1983). La dimensión oculta, Octava Edición. México: Editorial Siglo Veintiuno.

Knowles, M., Holton, F. y Swanson, R. (2001). Andragogía: el aprendizaje de los adultos. México: Mexicana.

Spearman, C. E. (1927). Las habilidades del hombre. Lóndres: McMillan.

Teixeira, A. (1956). A educación en al contexto brasileira. São Paulo: Compañía Editora Nacion.

\section{Sobre la autora}

\section{Regina Gabriela Chicas Sánchez}

Licenciada en Psicología, actualmente estudia la Maestría en Docencia Universitaria con Énfasis en Andragogía en el Centro Universitario de Suroriente CUNSURORI de la USAC. Se desempeña como profesora en la carrera de Licenciatura en Psicología del Centro Universitario de Zacapa CUNZAC -USAC.

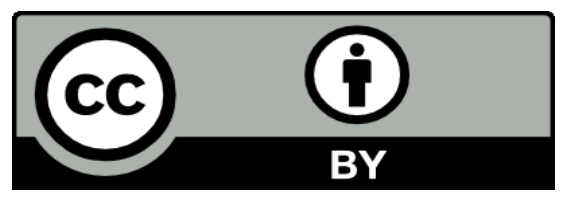

Este texto está protegido por una licencia CreativeCommons 4.0.

Usted es libre para compartir, copiar y redistribuir el material en cualquier medio o formato y adaptar el documento, remezclar, transformar y crear a partir del material para cualquier propósito, incluso comercialmente, siempre que cumpla la condición de atribución: usted debe reconocer el crédito de una obra de manera adecuada, proporcionar un enlace a la licencia, e indicar si se han realizado cambios. Puede hacerlo en cualquier forma razonable, pero no de forma tal que sugiera que tiene el apoyo del licenciante o lo recibe por el uso que hace. 